\title{
BLOOD PERFUSION OF SUBCUTANEOUS TUMOURS IN MICE FOLLOWING THE APPLICATION OF LOW-LEVEL DIRECT ELECTRIC CURRENT
}

\author{
T. Jarm, ${ }^{1}$ Y. A. B. D. Wickramasinghe, ${ }^{2}$ M. Deakin, ${ }^{2}$ M. Čemažar, ${ }^{3}$ \\ J. Elder, ${ }^{2}$ P. Rolfe, ${ }^{2}$ G. Serša, ${ }^{3}$ and D. Miklavčič ${ }^{1}$ \\ ${ }^{1}$ Faculty of Electrical Engineering \\ University of Ljubljana \\ Tržaška 25, SI-1000 Ljubljana \\ Slovenia. \\ ${ }^{2}$ School of Postgraduate Medicine, Keele University \\ Thornburrow Drive, Hartshill, Stoke-on-Trent, ST4 7QB \\ United Kingdom \\ ${ }^{3}$ Department of Tumour Biology \\ Institute of Oncology \\ Zaloška 2, SI-1000 Ljubljana \\ Slovenia
}

\begin{abstract}
Electrotherapy with low-level direct electric current has been proved to be an effective local treatment of solid tumours. In the presented study an attempt was made to evaluate the effect of a single treatment with electrotherapy on blood perfusion of solid subcutaneous fibrosarcoma Sa-1 tumours in $\mathrm{A} / \mathrm{J}$ mice. The tissue-staining method with Patent blue-violet dye, the rubidium extraction technique, and the noninvasive nearinfrared spectroscopy method were used for this purpose. Results of all methods indicate that perfusion and subsequently oxygenation of tumours were reduced due to application of electrotherapy.
\end{abstract}

\section{INTRODUCTION}

It has been shown in several studies by various authors that electrical current can be used for treatment of solid malignancies. Antitumour effectiveness of electrotherapy 
has been demonstrated on various experimental tumour models as well as in clinical trials (Humphrey and Seal, 1959; David et al., 1985; Marino et al., 1986; Heiberg et al., 1991; Griffin et al., 1994; Plesničar et al., 1994; Xin, 1994). In all reported studies electrotherapy was used as a local treatment and as such it can also be used as a suitable adjuvant treatment with other therapies in order to potentiate their localised effectiveness (Serša et al., 1992; Serša et al., 1993b). Many attempts have been made to try explain effectiveness of electrotherapy alone or of its combined use with other therapies. The understanding of the mechanisms of antitumour action is of importance for optimising existing and developing new treatment strategies. One can hardly expect that one single mechanism would be responsible for demonstrated antitumour effectiveness because wide ranges of different tumours and various treatment modalities were used in reported studies. In the present study we concentrate on one particular experimental tumour model which has been extensively used in the past in studies of the effects of direct current electrotherapy (DC-ET) alone and of its use in combination with certain anticancer drugs (Serša et al., 1992, 1993a, 1993b). Growth of murine fibrosarcoma Sa-1 tumours in A/J mice is temporarily retarded by application of a single DC-ET and tumours can be permanently eradicated when DC-ET is applied together with tumour necrosis factor TNF$\alpha$ at doses which are not effective when TNF- $\alpha$ alone is administered (Serša et al., 1994). Growth delay of treated tumours with respect to control tumours exhibits dose dependence on amplitude and duration of applied current (Serša et al., 1993a). The effects of different electrode materials and configurations on tumour growth have been studied as well as the effects of DC-ET on tissue $\mathrm{pH}$ and temperature, on production of tissue necrosis, and on electrode material deposition (Miklavčič et al., 1993, 1994). None of the above effects were found to be responsible for the demonstrated tumour growth retardation in the case when electrodes were placed outside the tumour with the tumour itself being located between the electrodes.

One of the proposed mechanisms of antitumour action of DC-ET for electrode configurations where tumour is not penetrated by the stimulation electrodes has been the effect of direct current on the vessels supplying the tumour at the site of electrode insertion (Griffin et al., 1995). Solid tumours in general are known to be hypoxic in comparison to normal tissues. Poor supply of oxygen and nutrients to tumours is a result of structurally and functionally abnormal microcirculation of blood in tumours. Deficient perfusion and oxygenation are not only limiting factors for tumour growth but can also result in resistance of tumours to radiotherapy and to treatment with various chemotherapeutics (Vaupel, 1979; Jain, 1988). Therefore it has been suggested that altering the blood supply to tumours by means of DC-ET might lead to eradication of tumour mass (Griffin et al., 1995).

In the presented work we assessed the effects of DC-ET on parameters of tumour perfusion by means of three methods: invasively by the tissue staining method using Patent blue-violet biological dye (PBV) and by the rubidium 86 extraction technique $\left({ }^{86} \mathrm{Rb}\right)$, and non-invasively using the optical method of near-infrared spectroscopy (NIRS).

\section{METHODS}

\subsection{Tumour Model}

Healthy female and male mice of immunocompetent $\mathrm{A} / \mathrm{J}$ strain (8-12 weeks of age) were used in the experiments. Only female animals were used for the near-infrared spectroscopy part of the study and animals of both sexes were used in experiments involving 
staining of tissue with Patent blue-violet (PBV) and rubidium extraction technique. Animals were purchased from Rudjer Bosković Institute, Zagreb, Croatia. Mice were kept in standard animal colonies and fed ad libitum. Solid subcutaneous tumours were initiated dorsolaterally in the right flank of animals with injection of saline suspension of about $5 \cdot 10^{5}$ viable fibrosarcoma Sa- 1 cells. Cells were obtained from ascitic form of Sa-1 tumours and their viability was assessed with standard trypane blue staining method. The experiments were performed 7 to 10 days after inoculation when tumours reached volume of about $50 \mathrm{~mm}^{3}$. Tumour volume was calculated from three mutually orthogonal diameters of the tumour $(a, b, c)$ measured with calliper and using the formula $V=$ $\pi a b c / 6$. The experiments were conducted in accordance with the European Council directives and the Slovenian and the British legislation concerning animal welfare.

\subsection{Electrotherapy (DC-ET)}

A single treatment with DC-ET consisted of one-hour treatment with a current of $0.6 \mathrm{~mA}$ amplitude. An adjustable current generator was used to provide regulated direct current which was delivered via a pair of subcutaneously inserted needle electrodes ( $\mathrm{Pt} / \mathrm{Ir}$ $90 / 10 \%$ alloy, diameter $1 \mathrm{~mm}$, length $2 \mathrm{~cm}$, rounded tip). Electrodes were inserted in parallel on two opposite sides of the tumour at least $5 \mathrm{~mm}$ away from its edge, with the anode (positive electrode) always on the caudal side of the tumour. Thus the tumour was not in physical contact with the electrodes (Figure 1). Animals in the control groups were treated in exactly the same way as animals subjected to DC-ET except that no current was applied. Throughout the experiments animals were lightly physically restrained and anaesthetised using the inhalation agent enflurane which was delivered in a mixture of oxygen and nitrous oxide. Body temperature was maintained at physiological values by a regulated heating pad on which the animals were placed.

\subsection{Tissue Staining with Patent Blue-Violet Dye (PBV)}

Saline solution of biological dye Patent blue-violet (Byk Gulden, Switzerland) was injected into the tail vein of animals (dose $0.2 \mathrm{ml}$, concentration $1.25 \%$ ). After the dye had been left to distribute evenly through the tissues for 2 minutes, animals were euthanised and tumours were removed from the skin and cut in half along their largest diameter. The percentage of stained area of tumour cross-section (perfused) as opposed to non-stained area (non-perfused) was visually estimated by two persons. An average of both estimations was used as an indicator of tumour perfusion. Experimental groups of 6 to 9 animals were formed with respect to the predefined time interval between the start of DC-ET and the moment at which animals were injected with the dye. Corresponding control groups were also formed (Szabo, 1985; Sagar et al., 1991).

\subsection{Rubidium Extraction Technique}

Relative tissue perfusion (RTP) was assessed by the ${ }^{86} \mathrm{Rb}$ extraction technique at predefined time intervals after DC-ET. For each time interval a separate group of animals was used with 4 to 6 animals per group. Corresponding control groups were also formed. For injection, a $50 \mathrm{mCi} / \mathrm{ml}$ solution of ${ }^{86} \mathrm{RbCl}$ (Amersham PLC, Little Chalfont, Bucks., UK) was used. At predefined intervals the $0.1 \mathrm{ml}$ aliquots of solution were injected into the tail vein of animals. Animals were euthanised one minute after injection. It has been experimentally verified that the amount of ${ }^{86} \mathrm{Rb}$ extracted by tissues one minute after injection was well within the plateau region required for validity of the method 
(Sapirstein, 1958; Zanelli and Fowler, 1974). The whole tumour, thigh muscle sample from the left hind leg, and the tail from the same animal were removed and placed in preweighed glass vials. Muscle samples from the leg were used for assessment of the effects of electrotherapy applied locally to the tumour in the right flank on perfusion of other tissues not directly exposed to electric current. All samples were weighed and their radioactivity was measured using a gamma counter (Institute Jožef Stefan, Ljubljana, Slovenia). In each measurement sequence the activity of three $0.1 \mathrm{ml}$ aliquots of injection solution and the background activity were also measured.

Radioactivity measured in the tumour and the muscle sample was corrected for the background activity and the dose radioactivity was corrected for the residual activity in the tail. All animals with more than $10 \%$ of the injected dose retained in the tail due to improper injection were excluded from further evaluation. The following equation was applied to calculate the RTP of tissues expressed as a percentage of dose extracted in the tissue sample normalised per sample weight:

$$
R T P(\% / \mathrm{g})=100 \cdot \frac{\left(\text { activity }_{S A M P L E}-\text { activity }_{B A C K G R O U N D}\right) / \text { weight }_{S A M P L E}}{\text { activity }_{D O S E}-\text { activity }_{T A I L}}
$$

\subsection{Near-Infrared Spectroscopy}

Two NIRO2-X2 instruments (Keele University, UK) were used in the study for simultaneous monitoring of concentration changes of oxygenated and deoxygenated haemoglobin ( $\mathrm{HbO}$ and $\mathrm{Hb}$ ) in tumour and normal reference tissue of the same animal. Pulsed laser near-infrared light was delivered to the tissue and collected from the tissue by two optical light guides (optodes). Concentration changes were calculated from changes in attenuation of light in tissue at three wavelengths $(904 \mathrm{~nm}, 845 \mathrm{~nm}, 775 \mathrm{~nm})$ using a modified Beer-Lambert law (Wickramasinghe et al., 1992). Two additional parameters were calculated from measured $\mathrm{HbO}$ and $\mathrm{Hb}$ changes; change of total haemoglobin concentration $\mathrm{Hb}$-tot defined as $\mathrm{HbO}+\mathrm{Hb}$ which is correlated with changes of blood volume in the tissue under observation, and change of differential haemoglobin concentration $\mathrm{Hb}$-dif defined as $\mathrm{HbO}-\mathrm{Hb}$. Monitoring of normal tissue (thigh muscles of left hind leg as opposed to tumour growing in the right flank) served for evaluation of general stability of the animal under anaesthesia and for assessing systemic responses to DC-ET. Before measurement all hair was removed from the skin covering the tumour and the muscle. Due to the small dimensions of the animals and tumours it was impossible to attach optodes to the site of measurement itself (Figure 1). Therefore both pairs of optodes were held fixed to desired positions by special holders.

Monitoring of NIRS parameters was started approximately 20 minutes after induction of anaesthesia. DC-ET was started only when stable NIRS signals from both tumour and muscle tissue had been recorded for at least 20 minutes. NIRS monitoring was maintained until approximately 60 minutes after the end of DC-ET and for a corresponding period in control animals.

\section{RESULTS}

\subsection{Tissue Staining with PBV Dye}

We used biological dye Patent blue-violet (PBV) instead of Evans blue which is otherwise a more widely used dye in perfusion studies. The reason for this is that the 


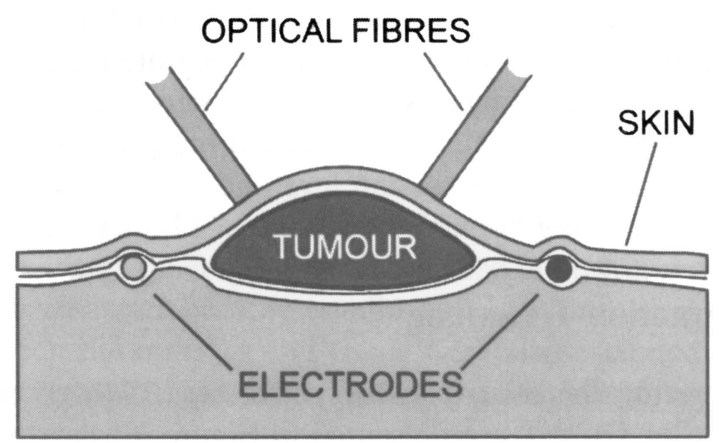

Figure 1. Position of electrodes for DC-ET and optical guides for NIRS measurements in tumours.

colour contrast between stained and non-stained areas of tissue was far better with PBV. We verified in a separate pilot study that both dyes produce virtually the same results for control tumours and for tumours treated with DC-ET (data not shown).

Results of staining the tumours with PBV at different intervals during and after ET are presented in Figure 2. The degree of staining of control tumours did not change during the period of observation and was about $90 \%$ of cross-section area. Five minutes after the onset of DC-ET tumours were still equally stained as the controls. Percentage of stained area then rapidly declined to minimum level of about $15 \%$ which was reached in 15-30 minutes. This reduced level was maintained for nearly 24 hours when some minor increase in perfusion could already be observed. Three days after DC-ET treat-



Figure 2. Perfusion of tumours at different time intervals during and after DC-ET assessed as percent of stained area of tumour cross-section. Mean values and standard error bars are shown ${ }^{*} \mathrm{P}<0.05,{ }^{* *} \mathrm{P}<0.001$; Student $\mathrm{t}$ test). 
ment the percentage of stained area reached about $60 \%$ which was still substantially less than in the corresponding control tumours. It should be noted that the results from individual tumours at different time intervals were very homogeneous in all control groups. However, scattering of individual data was increased for DC-ET-treated tumours especially during dynamic changing of perfusion level, i.e. during "deperfusion" in the first half hour of DC-ET and during "reperfusion" 24 to 72 hours after DC-ET.

\subsection{Rubidium Extraction Technique}

Results of measuring the relative tissue perfusion (RTP) of tumours at different intervals during and after DC-ET are presented in Figure 3.

As soon as 15 minutes after the start of application of electrotherapy, the RTP of the tumours had already reached the minimum level of about $1.5 \% / \mathrm{g}$ which was more than a $50 \%$ reduction with respect to control tumours where RTP was about $3.5 \% / \mathrm{g}$. A statistically significant decrease in RTP was maintained for two days except for the group of tumours 24 hours after treatment. The mean value of these tumours was surprisingly high but this was due to three tumours which were apparently not successfully treated. Three days after treatment a partial reperfusion of treated tumours was demonstrated. Control tumours on their own exhibited a gradual decrease in perfusion with increasing size and age of tumours as expected. As with the staining technique, the scattering of results of individual animals was higher for DC-ET than for control animals.

No significant change due to the application of DC-ET to tumours was observed in thigh muscles which were not directly exposed to electrical treatment. Perfusion of muscles also did not change significantly during the observation period of three days.

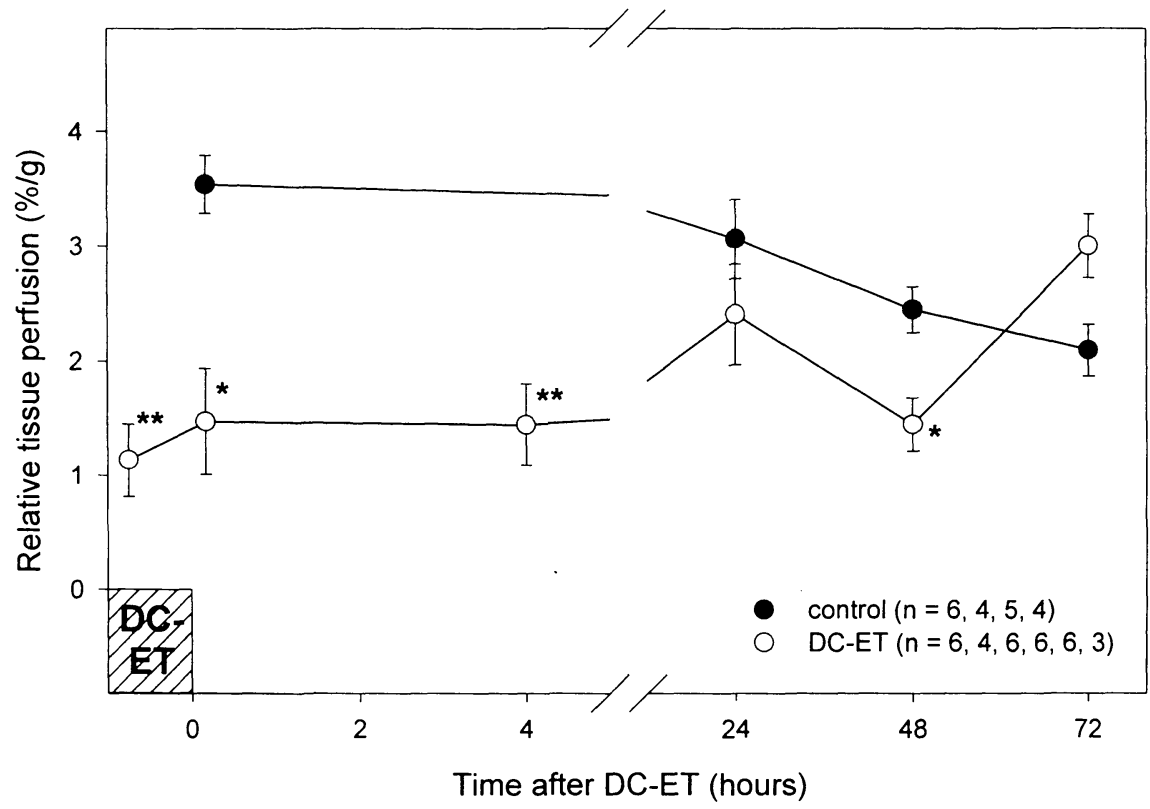

Figure 3. Relative tissue perfusion of tumours at different time intervals during and after DC-ET assessed by the rubidium extraction technique. Mean values and standard error bars are shown $\left({ }^{*} \mathrm{P}<0.05,{ }^{* *} \mathrm{P}<0.001\right.$; Student $\mathrm{t}$ test). 
The mean RTP values of muscles in all treated and control animals were within the $3-4 \% / g$ range (data not shown).

\subsection{Near-Infrared Spectroscopy}

Only those animals were included in evaluation for which stable baseline values of measured parameters prior to therapy were established in both tumour and normal tissue. All cases where measurements became invalid during monitoring due to disruption of optical contact between the optodes and tissue were also excluded from further evaluation. Total rejection rate was about $30 \%$. Thus 5 and 6 animals remained in DC-ET and control group, respectively.

There was a large variability in magnitudes of response of the measured parameters to DC-ET between individual tumours. Nevertheless, common typical features were observed in the majority of tumours.

Figure 4 presents data recorded from two typical tumours from DC-ET and control groups. Onset of DC-ET resulted in mild transient excitation of the animals despite anaesthesia. After initial excitation $\mathrm{HbO}$ decreased rapidly to a level lower than before DC-ET. This level was maintained for a period of about 30 minutes in most animals but then $\mathrm{HbO}$ started to increase gradually. Immediately following the excitation $\mathrm{Hb}$ started to increase thus partially matching the rapid decrease of $\mathrm{HbO}$. After the minimum $\mathrm{HbO}$ level had been reached $\mathrm{Hb}$ continued to increase at a lesser but steady rate. $\mathrm{Hb}$-tot was initially decreased due to the rapid decrease of $\mathrm{HbO}$ but then it started to increase due to the increasing $\mathrm{Hb}$ and later also due to the increasing $\mathrm{HbO}$. Hb-dif decreased in all treated tumours without exception and in most tumours it continued to decrease during the whole period of observation. In tumours of control animals and also in muscles of


Figure 4. Typical NIRS measurements of individual response of control tumour (a) and of tumour treated with DC-ET (b). 

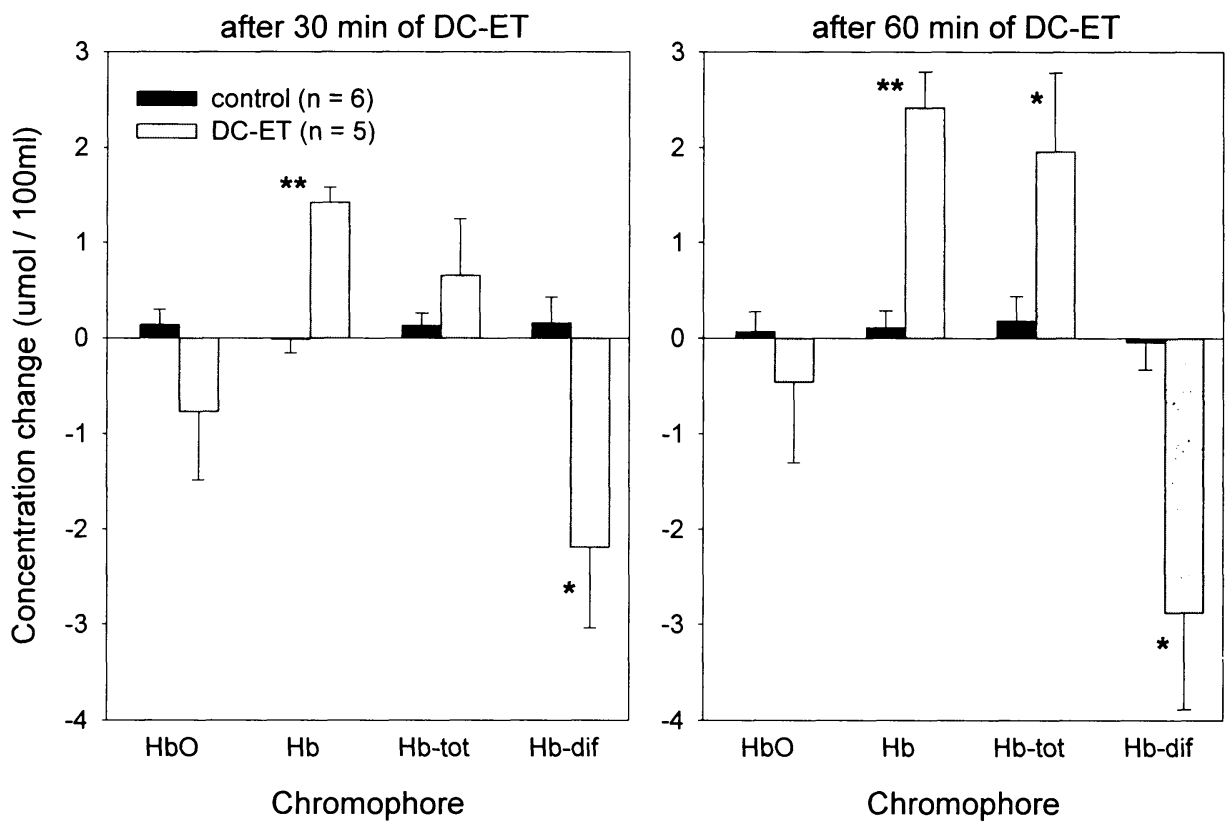

Figure 5. Comparison of changes in concentration of NIRS parameters ( $\mathrm{HbO}, \mathrm{Hb}, \mathrm{Hb}-\mathrm{tot}, \mathrm{Hb}-\mathrm{dif})$ in control tumours and tumours treated with DC-ET. Mean values and standard error bars are shown $\left({ }^{*} \mathrm{P}<0.05,{ }^{* *} \mathrm{P}<\right.$ 0.001 ; Student $\mathrm{t}$ test).

DC-ET-treated animals there were no significant changes of measured parameters. Average changes of measured tumour parameters in both experimental groups at two characteristic time intervals with respect to the moment of start of DC-ET are presented in Figure 5.

\section{DISCUSSION AND CONCLUSIONS}

The perfusion data obtained with the tissue staining method and by the rubidium extraction technique clearly demonstrate that blood supply to the tumours was severely decreased due to application of DC-ET. Although the direct histological evidence is not available at the moment, we suggest that the most probable reason for this was damaged blood vessels and subsequent vascular occlusion at the site of insertion of the electrodes especially since the skin between the electrodes and the tumour was also not stained in most cases and the skin where the electrodes were inserted appeared macroscopically damaged. Extreme $\mathrm{pH}$ changes as the result of electrolysis in the immediate vicinity of the electrodes demonstrated by (Miklavčič et al., 1993) could explain this effect. In the samples taken during the "deperfusion" period (first 30 minutes after the start of ET) we observed that non-perfused areas in tumours occurred first in the part of tumour that was closer to the cathode. This is in accordance with the results of previous studies where it was demonstrated that post-treatment necrosis at the site of insertion of the electrodes was more extensive at the cathode than at the anode in both tumour and normal tissue. Rapid decrease in $\mathrm{HbO}$ and $\mathrm{Hb}$-tot after the start of DC-ET as demonstrated by NIRS in the majority of treated tumours correlates well with the perfusion data. The dynamics of the 
decrease in PBV staining and $\mathrm{HbO}$ are very similar. Maximum decrease in $\mathrm{HbO}$ and minimum level of tissue staining are reached on average in about 15 minutes and in 15-30 minutes respectively after onset of DC-ET. Faster decrease of $\mathrm{HbO}$ could be explained by increased extraction of oxygen from the blood (deoxygenation of haemoglobin) which would occur in case of decreased blood flow through tumour. This hypothesis is supported by the fact that $\mathrm{Hb}$ was increasing more rapidly during decreasing of $\mathrm{HbO}$ than at the latter stage after the minimum $\mathrm{HbO}$ level had been reached which is an indicator of increased rate of deoxygenation of haemoglobin. The slow but steady increasing of $\mathrm{Hb}$ and $\mathrm{Hb}$-tot observed at latter times indicates that blood volume was increasing. This could be explained if venous outflow from the tumour was more severely impaired than arterial inflow, for which however, no direct evidence is available at the moment. This would mean that fresh blood was still able to enter some parts of the tumour in reduced quantities but it was not able to leave the tumour. This would lead to gradual accumulation of blood (increasing of $\mathrm{Hb}$-tot) in the tumour. We observed substantial quantities of spilled blood within some of tumours treated with DC-ET and in their immediate vicinity. This effect was absent in control tumours. We think that the large $\mathrm{Hb}$-tot increase at the end of NIRS monitoring accounts for this blood pooling. The moment when $\mathrm{HbO}$ started to increase in some tumours (but not in all) probably indicated when leaking from vessels in the tumours began. It is however possible that the recorded NIRS signals also contained contributions from the tissue surrounding the tumours due to the small size of tumours.

The reperfusion dynamics in the days following the DC-ET treatment as demonstrated by tissue staining and rubidium extraction (Figures 2,3 ) were in good agreement with known dynamics of regrowth of Sa-1 tumours after DC-ET (Serša et al., 1993a). On average in the first days following DC-ET the size of these tumours was reduced. Then 3 to 4 days after treatment tumours started growing again. Tissue staining with PBV and rubidium extraction revealed that 3 days after treatment partial (PBV) or total (rubidium) reperfusion occurred.

We have previously observed that not all tumours are equally responsive to DCET resulting in wider ranges of values of all measured parameters for DC-ETtreated tumours in comparison to control tumours. In the light of the effect of DC-ET on tumour-feeding vessels the main possible factor besides the biological differences between tumours would be the relative proximity of these vessels to electrodes during treatment and the ratio of affected and unaffected supplying vessels.

Measurements taken from normal muscle tissue in case of NIRS and rubidium extraction technique confirmed our expectations that the effects of DC-ET were indeed localised in the area between the electrodes and that DC-ET did not produce significant perfusion changes in other tissues.

We conclude that perfusion and consequently oxygenation of subcutaneous Sa-1 fibrosarcoma tumours in $\mathrm{A} / \mathrm{J}$ mice were significantly decreased due to electrotherapy. The results obtained by means of all three methods are mutually supportive and are in good agreement with known growth dynamics of tumours after treatment. In this particular tumour model the effect on tumour blood supply is most probably the primary mechanism of antitumour action of DC-ET.

\section{ACKNOWLEDGMENT}

The studies were supported by the Ministry of Science and Technology of the Republic of Slovenia and by the EC Concerted Action Project on Near Infrared 
Spectrophotometry and Imaging of Biological Tissue (funded by Commission of the European Communities, Directorate-General for Science, Research and Development, International Scientific Cooperation, Brussels, Belgium).

\section{REFERENCES}

David SL, Absolom DR, Smith CR, Gams J, and Herbert MA (1985) Effect of low level direct current on in vivo tumor growth in hamsters. Cancer Res. 45:5626-5631.

Griffin DT, Dodd NJF, Moore JV, Pullan BR, and Taylor TV (1994) The effects of low-level direct current therapy on a preclinical mammary carcinoma: tumour regression and systemic biochemical sequelae. Br. J. Cancer 69:875-878.

Griffin DT, Dodd NJF, Zhao S, Pullan BR, and Moore JV (1995) Low-level direct electrical current therapy for hepatic metastases. I. Preclinical studies on normal liver. Br. J. Cancer 72:31-34.

Heiberg E, Nalesnik WJ, and Janney C (1991) Effects of varying potential and electrolytic dosage in direct current treatment of tumors. Acta Radiol. 32:174-177.

Humphrey CE and Seal EH (1959) Biophysical approach towards tumor regression in mice. Science 130:388-390.

Jain RK (1988) Determinants of tumor blood flow: a review. Cancer Res. 48:2641-2658.

Marino AA, Morris D, and Arnold T (1986) Electrical treatment of Lewis lung carcinoma in mice. J. Surg. Res. 41:198-201.

Miklavčič D, Serša G, Kryžanowski M, Novaković S, Bobanović F, Golouh R, and Vodovnik L (1993) Tumor treatment by direct electric current - tumor temperature and $\mathrm{pH}$, electrode material and configuration. Bioelectroch. Bioenerg. 30:209-220.

Miklavčič D, Fajgelj A, and Serša G (1994) Tumor treatment by direct electric current: electrode material deposition. Bioelectroch. Bioenerg. 35:93-97.

Plesničar A, Serša G, Vodovnik L, Jančar J, Zaletel-Kragelj L, and Plesničar S (1994) Electric treatment of human melanoma skin lesions with low level direct electric current: an assesment of clinical experience following a preliminary study in five patients. Eur. J. Surg. Suppl. 574:45-49.

Sagar KB, Pelc LR, Rhyne TL, Howard J, and Warltier DC (1991) Estimation of myocardial infarct size with ultrasonic tissue characterization. Circulation 83:1419-1428.

Sapirstein LA (1958) Regional blood flow by fractional distribution of indicators. Am. J. Physiol. 193:161-168.

Serša G, Miklavčič D, Batista U, Novaković S, Bobanović F, and Vodovnik L (1992) Anti-tumor effect of electrotherapy alone or in combination with interleukin-2 in mice with sarcoma and melanoma tumors. Anti-Cancer Drugs 3:253-260.

Serša G and Miklavčič D (1993a) The feasibility of low level direct current electrotherapy for regional cancer treatment. Reg. Cancer Treat. 1:31-35.

Serša G, Novaković S, and Miklavčič D (1993b) Potentiation of bleomycin antitumor effectiveness by electrotherapy. Cancer Lett. 69:81-84.

Serša G, Golouh R, and Miklavčič D (1994) Anti-tumor effect of tumor necrosis factor combined with electrotherapy on mouse sarcoma. Anti-Cancer Drugs 5:69-74.

Szabo G (1985) New steps in the intra-arterial chemotherapy of head and neck tumors. Oncology 42:217-223.

Vaupel P (1979) Oxygen supply to malignant tumors. In Tumor Blood Circulation: Angiogenesis, Vascular Morphology and Blood Flow of Experimental and Human Tumors (Peterson H-I, Ed.) CRC Press, Boca Raton, pp. 143-168.

Wickramasinghe YABD, Livera LN, Spencer SA, Rolfe P, and Thorniley MS (1992) Plethysmographic validation of near infrared spectroscopic monitoring of cerebral blood volume. Arch. Dis. Child. 67:407-411.

Xin Y-L (1994) Advances in the treatment of malignant tumours by electrochemical therapy. Eur. J. Surg. Suppl 574:31-36.

Zanelli GD and Fowler JF (1974) The measurement of blood perfusion in experimental tumors by uptake of ${ }^{86}$ Rb. Cancer Res. 34:1451-1456. 